\title{
Acercamiento al cálculo de índice de salud de transformadores de fuerza en Cuba
}

\section{An approach on calculation of Health Index of power transformers in Cuba}

Jorge Juan Montané-García ${ }^{1}$, Orestes Nicolás Hernández-Areu², Alfredo Manuel del-Castillo-Serpa ${ }^{3}$

Montané-García, J.J; Hernández-Areu, O.N; del-CastilloSerpa, A.M. Acercamiento al cálculo de índice de salud de transformadores de fuerza en Cuba. Tecnología en Marcha. Vol. 34, especial. ALTAE. Diciembre 2021. Pág 34-47.

doi) https://doi.org/10.18845/tm.v34i7.6010

1 Unión Nacional Eléctrica, Central Termoeléctrica "Ernesto Guevara de la Serna", Cuba. Correo electrónico: jorgemon@ctehabana.une.cu (iD https://orcid.org/0000-0002-9390-1937

2 Universidad Tecnológica de la Habana "José A. Echeverría", Facultad de Ingeniería Eléctrica, Centro de Investigaciones y Pruebas para la Electricidad (CIPEL), Cuba. Correo electrónico: orestesh@electrica.cujae.edu.cu (iD https://orcid.org/0000-0002-2672-239X

3 Universidad Tecnológica de la Habana "José A. Echeverría", Facultad de Ingeniería Mecánica, Centro de Estudios e Investigaciones del Mantenimiento (CEIM), Cuba. Correo electrónico: acastillo@cemat.cujae.edu.cu 


\title{
Palabras clave
}

Transformadores de fuerza; envejecimiento; aislamiento; índice de salud; diagnóstico.

\section{Resumen}

Los transformadores de fuerza (TF) son parte principal de los costos de un sistema electroenergético (SE), por lo que debe controlarse su estado, envejecimiento y vida remanente, para valorar su condición actual y futura, algo importante para tomar decisiones de operación, mantenimiento o inversiones, favoreciendo el uso óptimo de las finanzas y asegurando la confiabilidad y calidad. Un fallo de aislamiento de un TF implica daños sensibles, genera altos costos de reparación y pérdidas financieras. Al sistema de aislamiento papel-aceite del TF es imposible realizarle mantenimientos, por lo que debe evaluarse su condición, para prevenir posibles defectos de los TF, minimizando el riesgo de fallas y salidas de servicio. No es factible, desde el punto de vista económico, someter TF envejecidos a inspecciones y ensayos rigurosos. En este caso, una estrategia promisoria para elevar el tiempo de vida remanente de estos equipos lo constituye el establecimiento de prioridades de monitoreo y el desarrollo de un plan detallado de mantenimiento. He aquí el por qué juegan un papel primordial los sistemas de monitoreo y diagnóstico del aislamiento. El Índice de Salud (IS) se utiliza para cuantificar el estado global de los TF, tomando en consideración una multitud de factores. En el trabajo se realiza un acercamiento al cálculo del IS de TF en las condiciones de una central termoeléctrica (CTE) de Cuba, donde, debido a las condiciones económicas imperantes, no es posible realizar la medición de todos los factores de diagnóstico utilizados en otros países con este fin.

\section{Keywords}

Power transformers; ageing; isolation; health index; diagnostics.

\begin{abstract}
Power transformers (PT) are a main part of costs of electro energetic systems (EES), so it must to control its state, ageing and remnant life, in order to assess its present and future condition, which is important to take correct decisions in operation, maintenance or investments, for assure the financial optimization, the reliability and the quality of all the EES. A fault in the isolation system of a PT carry out sensitive damages, generates high repair costs and financial lost. It is impossible to execute maintenances in the paper-oil isolation system of the PT, so it must to assess its condition, in order to prevent possible faults of the PT, minimizing the risk of failures and outages. It is not economic for an old transformer to be subjected to rigorous inspections and thorough testing. A promising strategy for lifetime increase is to establish monitoring priorities and develop a strategy for their maintenance. This is why insulation systems monitoring and diagnosis has become an important part in transformers monitoring. The Health Index $(\mathrm{HI})$ is used to quantify the global state of the PT, taking into account a multitude of factors. In this paper, authors make an approah to the calculation of the HI of the PT in a termal power station (TPS) in Cuba, where, doing of the actual eonomic conditions, it is not possible to execute the meassurements of all the diagnostic fators, which are used in another countries.
\end{abstract}

\section{Introducción}

Por su gran volumen, las reparaciones en los TF son muy complejas y costosas, por lo que debe evitarse su realización [1]; por ello, la determinación confiable de la vida remanente de los TF es de suma importancia, tanto para las empresas energéticas como para los 
consumidores [2]. Diagnosticar la condición de los TF es fundamental para su gestión de vida. No es económicamente factible someter TF envejecidos a inspecciones y ensayos rigurosos, por lo que deben establecerse prioridades de monitoreo y desarrollarse un plan detallado de mantenimiento, en lo que juegan un papel primordial los sistemas de monitoreo y diagnóstico del aislamiento, basados en una amplia gama de ensayos físicos, eléctricos, mecánicos, térmicos y en comprobaciones visuales, que permiten evaluar el estado de los TF, proveen información sobre su envejecimiento y recomiendan medidas para mejorar la calidad del aislamiento y la evaluación del tiempo de vida [3]. Los TF están sometidos continuamente a esfuerzos de envejecimiento térmicos, eléctricos, ambientales, mecánicos, químicos y otros $[4,5]$, que ocasionan su deterioro al transcurrir el tiempo de operación (TO), además de que pueden experimentar esfuerzos extremos y daños internos de sus componentes principales, debidos a fallas externas [4, 6]. Los TF se componen de un núcleo, aceite dieléctrico y papel (celulosa), que constituye el aislamiento fundamental de los equipos, cuyo trabajo y tiempo de vida remanente están en dependencia del estado del papel impregnado en aceite. Es posible cambiar el aceite, pero el cambio de aislamiento sólido del TF es tan costoso que es preferible adquirir un equipo nuevo; es decir, el fin de la vida útil del papel implica el fin de la vida útil del TF (por lo general alrededor o más de 40 años) [2]. Como al aislamiento papel-aceite es imposible realizarle mantenimientos, es necesario evaluar su condición, para minimizar el riesgo de fallas y salidas de servicio; de ahí la importancia de evaluar la probabilidad de vida remanente de los TF. El conocimiento de la vida remanente de los TF es decisivo para administrar el riesgo asociado con la confiabilidad de la red y, por supuesto, para brindar un servicio eléctrico de calidad [4].

En la evaluación de activos es muy popular el IS, para determinar el cual han sido utilizadas las variantes de "pesaje" y "ponderación", complementadas con la lógica difusa, redes neuronales y otras herramientas de la Inteligencia Artificial (IA). EI IS permite, mediante el procesamiento de la información disponible sobre los TF, realizar una evaluación general de su condición, sobre la base de los resultados de las condiciones de campo de los equipos, los resultados de inspecciones, ensayos in situ y otros [7].

El reemplazo de TF, sobre la base del TO, ha sido en la actualidad prácticamente desechado $[4,8]$. La vida técnica remanente de los TF debe determinarse sin utilizar métodos que requieran de costoso equipamiento, sino con el tratamiento y análisis estadístico de datos y análisis de riesgo, lo que permitirá tomar decisiones relativas a la ejecución de mantenimientos o reemplazo de los TF. Es necesario encontrar una variante que permita utilizar la información disponible de explotación, mantenimiento y los datos obtenidos de los controles e inspecciones de diagnóstico, considerando varios factores de degradación [4]. Para calcular el tiempo de vida remanente de los TF es necesario primeramente realizar un cálculo de su IS, lo que, en las condiciones de Cuba, con severos problemas económicos, que impiden o retrasan la adquisición de equipamiento de diagnóstico, se dificulta, por lo que es necesario comprobar si los métodos propuestos internacionalmente, que implican el tratamiento matemático de la información disponible sobre los TF, son utilizables en las condiciones de nuestro país. Este aspecto, que los autores desconocen se haya tratado con anterioridad, constituye el objetivo principal del presente trabajo.

\section{Índice de Salud del transformador de fuerza y su determinación}

EI IS es una medida de la condición de los activos, no determinada fácilmente mediante inspección rutinaria. Mientras las condiciones de funcionamiento empeoran, la probabilidad de falla aumenta, siendo conveniente clasificar los activos en función de su condición técnica, en base a los ensayos de diagnóstico que se realicen, lo que es útil cuando el número de activos 
es considerable. El IS es una puntuación asignada a cada activo, que clasifica su condición y mide su salud global, permitiendo compararlo con el resto e identificar en cuáles es necesario tomar acciones a corto, mediano y largo plazo. No existe un método único para obtener el IS, además de que su nivel de dificultad puede variar, a causa de las pruebas que se utilicen como datos de entrada y del procedimiento de cálculo [9]. El IS se obtiene a partir de los resultados de ensayos del equipo, que dan información sobre algún aspecto de su condición, que permite establecer una calificación en función de su condición técnica [10] y tiene relación directa con la degradación o envejecimiento, proporcionando un valor cuantificado, que permite jerarquizar los equipos y tomar decisiones de gestión sobre cada uno de ellos. Para determinar el IS de un TF, se considera, como elemento dominante, el aislamiento aceite-papel. El IS es útil para representar la gravedad y cuantificar los diversos parámetros que afectan directa 0 indirectamente las características de envejecimiento y funcionamiento del TF [11].

Las empresas energéticas prestan mayor atención a la condición de salud de grupos de TF o de unidades que exhiban el menor IS, por lo que el IS es una herramienta efectiva para determinar la condición de los equipos [12]. Combinando el monitoreo de los datos obtenidos de las mediciones tipo, ensayos de rutina, historial de mantenimiento y estadística de fallas y sobre la base del criterio de expertos, puede realizarse una evaluación suficientemente abarcadora de la condición de salud del TF. Partiendo del IS de un grupo de TF, puede desmarcarse uno en la flota y juzgar si su condición se encuentra por debajo o por encima del IS promedio, aplicando la técnica estadística de regresión de los resultados del IS, lo que provee al personal de una referencia acerca de que debe prestarse mayor atención a TF con una condición de salud menor del IS promedio [12]. Es importante que el IS se tome como un parámetro variable, cambiante durante toda la vida útil del equipo [13].

\section{Parámetros para el cálculo del Índice de Salud}

El IS compuesto es muy utilizado para representar la condición general de un activo, cuantificando su condición en numerosos criterios, relativos a los factores de degradación a largo plazo, que al acumularse llevan el equipo a su fin de vida [14].

Para determinar el estado técnico de los TF, se realizan una gran cantidad de pruebas, enfocadas en sus diferentes componentes: aceite, aislamiento sólido, devanados, circuito magnético, etc. La condición del TF, según su IS, se divide en cinco categorías (muy bueno, bueno, favorable, pobre y muy pobre), atendiendo a la predicción del tiempo de vida útil y el nivel de degradación de sus componentes. El análisis de los gases disueltos (AGD), de la calidad del aceite y el contenido de furanos se usan para detectar los tipos de fallas que podrían ocurrir en el TF y el resultado del análisis son las predicciones de tiempos de vida, tipos de posibles fallas y recomendaciones de mantenimiento. Ya calculado el IS, se recomiendan tres tipos de acciones sobre los TF; sobre los que están trabajando normalmente, sobre los que continúan trabajando con restricciones y sobre los que requieren nuevas pruebas de muestreo. Finalmente, se concluye que, si bien el IS no puede conocer el tipo de fallas que ocurrieron en los TF, sí proporciona la condición general de salud de los mismos [15].

Existen tres requerimientos básicos para desarrollar el IS: las entradas, los algoritmos y las salidas.

Las entradas varían entre las cantidades, medidas regularmente, como parte del mantenimiento de rutina, como son [7, 10, 14]: resultados de ensayos de calidad del aceite; resultados del Análisis de Gases Disueltos en el aceite (AGD); condición general del TF, edad e historial de carga; inspecciones visuales de rutina del tanque, comprobando la existencia o no de salideros de aceite, su nivel, el estado de los radiadores, ventiladores, bushings, juntas y demás 
accesorios; resultados de inspecciones y ensayos del conmutador de elementos; resultados de inspecciones y ensayos de bushings; resistencia de los devanados a la corriente directa (CD); relación de transformación; resistencia de devanados y resistencia de aislamiento.

Los algoritmos varían entre el promedio de ponderación, el puntaje logarítmico, el acercamiento escalonado, el puntaje por grupos, el indexado de subconjuntos, la lógica difusa, redes neuronales y otros [7].

Las salidas pueden construirse de distintas formas. En el cuadro 1 se muestra la evaluación de la condición de degradación de TF según su IS.

Cuadro 1. Evaluación de la condición de degradación de TF según su IS.

\begin{tabular}{|c|c|c|}
\hline IS & Condición & Recomendaciones \\
\hline $0,85<\mathrm{IS} \leq 1,0$ & Muy buena & Mantenimiento normal \\
\hline $0,7<\mathrm{IS} \leq 0,85$ & Buena & Mantenimiento normal \\
\hline $0,5<\mathrm{IS} \leq 0,7$ & Fallado & Incrementar ensayos de diagnóstico \\
\hline $0,3<\mathrm{IS} \leq 0,5$ & Pobre & Comenzar plan de reparación o reconstrucción \\
\hline $\mathrm{IS} \leq 0,3$ & Muy pobre & $\begin{array}{c}\text { Evaluar inmediatamente el riesgo; cambiar o reconstruir, según indique la } \\
\text { evaluación }\end{array}$ \\
\hline
\end{tabular}

Fuente:[16]

La gestión de una flota de TF envejecidos es un desafío para sus propietarios. El aislamiento liquido de los TF, usualmente aceite mineral, se oxida, generándose ácidos, humedad y otros componentes polares, como alcoholes e hidroperóxidos, que forman compuestos insolubles en el aceite ("lodos"). El aislamiento sólido, como el papel y el cartón, se somete inicialmente a oxidación, hidrólisis y otros procesos, generándose humedad, ácidos, monóxido y dióxido de carbono, furanos y metanol [17]. La evaluación del envejecimiento de TF ha adquirido relevancia, debido a la elevación de los requerimientos de confiabilidad y longevidad de los equipos, que han permanecido en servicio un mayor número de años que el total de su vida operacional planificada. Con el transcurso del TO, se producen cambios en las propiedades del aislamiento del TF. Como al aceite de los TF se puede acceder con facilidad y el mismo contiene productos de la degradación, tanto del aislamiento sólido como de la del aceite en cuestión, la evaluación del envejecimiento comprende fundamentalmente la ejecución de ensayos del aceite y la interpretación de sus resultados. Una referencia común para la interpretación de los resultados de los ensayos del aceite la constituye la norma IEC $60422[17,18]$.

\section{Cálculo del Índice de Salud de transformador de fuerza}

Para evaluar la condición general de un TF se toman en cuenta todos los datos en existencia sobre su comportamiento, es decir se realizar un examen multifactorial. Cada uno de los componentes que influyen en la condición del TF debe examinarse por separado. El valor final del IS se calcula en base a la fórmula $[3,14,16]$ :

$$
I S=A 1 \times \frac{\sum_{i=1}^{n-3} K i F I S i}{\sum_{i=1}^{n-3} 4 K i}+A 2 \times \frac{\sum_{i=n-3}^{n} K i F I S i}{\sum_{i=n-3}^{n} 4 K i}
$$




\section{Donde [14]}

- Los factores de peso A1 y A2, correspondientes, respectivamente, al TF y a su cambiador de derivaciones, se toman como $60 \%$ y $40 \%$, sobre la base de una encuesta internacional llevada a cabo por el grupo de CIGRÉ que atiende las fallas en grandes TF.

- Ki es un coeficiente que se otorga a cada componente según su importancia dentro del TF en general;

- FISi es el Índice de Salud que se calcula para cada componente por separado.

Cada TF debe registrarse en una base de datos (BD) y, con la información de ensayos registrados, se calcula su IS. El sistema para el cálculo debe también desplegar la información de evolución de los IS en el tiempo, listado de IS de TF y recomendaciones basadas en criterios normativos o definidos con el personal de explotación. La herramienta debe generar reportes por TF; de igual forma, toda la información registrada puede ser guardada por el usuario para obtener información adicional de los TF. El sistema debe evaluar la condición de los TF que se incluyan, permitiendo registrar información por interoperabilidad con otras aplicaciones, registrar resultados de ensayos de TF, calcular el IS total de cada TF, generar una puntuación de todos los TF según su IS, analizar posibles fallas y elaborar recomendaciones de acciones a seguir. La herramienta también debe permitir observar la evolución del IS, para realizar un seguimiento de la condición de cada TF en el tiempo, de acuerdo a la frecuencia con que sean realizados los ensayos [10].

El cuadro 2 muestra los datos del TF de mayor edad en la flota que se estudia, al cual se le realizará el cálculo de su IF.

\section{Análisis de Gases Disueltos}

El Análisis de Gases Disueltos (AGD) es un método de diagnóstico frecuentemente usado para evaluar la condición interna del TF, pues mediante el estudio de la evolución de los gases, sus combinaciones, tendencias y valores individuales, puede relacionarse si el contenido de gases responde a mecanismos naturales de envejecimiento o determinar si existe una falla emergente [9]. La norma IEC 60599 [19] provee una lista de las fallas detectables por el AGD, mientras la norma IEEE Std C57.104 ${ }^{\mathrm{TM}}$ [20] introduce el criterio de cuatro niveles para clasificar los riesgos en los TF si se les mantiene una operación continua con los volúmenes de gases combustibles en el aceite detectados [14].

Teniendo en cuenta diferentes recomendaciones, el cuadro 3 y la figura 1 introducen un método de puntuación que utiliza la información suministrada por el AGD.

El factor de AGD se calcula como $[3,14]$ :

$$
F A G D=\frac{\sum_{i=1}^{7} S i \times W i}{\sum_{i=1}^{7} W i}
$$

Donde $[3,14]$ :

- Wi: factor de ponderación asignado, con valores iniciales de 1 para $\mathrm{CO}$ y $\mathrm{CO}_{2}$; 2 para $\mathrm{H}_{2}$; 3 para $\mathrm{CH}_{4}, \mathrm{C}_{2} \mathrm{H} 6$ y $\mathrm{C}_{2} \mathrm{H}_{4}$ y 5 para $\mathrm{C}_{2} \mathrm{H}_{2}$.

- $\mathrm{Si}=1,2,3,4,5$ o 6: puntuación de cada gas sobre la base de la figura 1; si el contenido de un gas excede el último limite introducido en la misma, se asigna un valor de 6.

Los códigos que se obtienen comienzan con A, como mejor condición y terminan en E, la peor. Este tipo de codificación es empleada para los factores restantes [14]. 
Cuadro 2. Datos de transformador para cálculo del IS.

\begin{tabular}{|c|c|c|c|c|c|c|c|c|c|c|c|}
\hline \multicolumn{9}{|c|}{ Gases disueltos, ppm } & \multicolumn{3}{c|}{ Características dieléctricas del aceite aislante } \\
\hline $\begin{array}{c}\text { Años de } \\
\text { servicio }\end{array}$ & $\mathrm{H}_{2}$ & $\mathrm{CH}_{4}$ & $\mathrm{C}_{2} \mathrm{H}_{2}$ & $\mathrm{C}_{2} \mathrm{H}_{4}$ & $\mathrm{C}_{2} \mathrm{H}_{6}$ & $\mathrm{CO}$ & $\mathrm{CO}_{2}$ & $\begin{array}{c}\text { Agua, } \\
\text { ppm }\end{array}$ & $\begin{array}{c}\text { Tensión de } \\
\text { ruptura, } \mathrm{KV}\end{array}$ & $\begin{array}{c}\text { Tensión } \\
\text { interfacial } \\
(\mathrm{TIF}), \text { dinas/cm }\end{array}$ & $\begin{array}{c}\text { Acidez, } \\
\mathrm{mg} \\
\mathrm{KOH} / \mathrm{g}\end{array}$ \\
\hline 11 & 0,5 & 0 & 107,52 & 369,05 & 44,48 & 2587,69 & 25870,51 & 16,43 & 60 & 43,94 & 0,0367 \\
\hline 14 & & & & & & & & 16,66 & 60 & 44,39 & 0,0272 \\
\hline 15 & & & & & & & & 32,6 & 46,8 & 41,33 & 0,0627 \\
\hline 17 & & & & & & & & 8 & 60 & 44,42 & 0,015 \\
\hline 18 & 7 & 13 & 2 & 128 & 19 & 75 & 1365 & 8 & 60 & 45,8 & 0,0148 \\
\hline 19 & 5 & 2 & 2 & 4 & 2 & 162 & 1589 & 8 & 60 & 57,6 & 0,0065 \\
\hline 21 & 5 & 2 & 10 & 13 & 2 & 146 & 2788 & 15 & 60 & 59,14 & 0,0097 \\
\hline 22 & & & & & & & & 13 & 60 & 54,35 & 0,0103 \\
\hline 22 & 6 & 3 & 2 & 24 & 2 & 114 & 2806 & 19 & 60 & 58,52 & 0,0092 \\
\hline 22 & 5 & 3 & 2 & 14 & 2 & 153 & 2367 & 14 & 60 & 56,49 & 0,0047 \\
\hline 23 & 5 & 3 & 17 & 19 & 2 & 116 & 2593 & 14 & 59,8 & 48,41 & 0,0091 \\
\hline 23 & 9 & 2 & 20 & 20 & 2 & 129 & 2558 & 19 & 75,4 & 64,11 & 0,0084 \\
\hline 24 & 6 & 2 & 20 & 19 & 2 & 108 & 2308 & 13 & 91,4 & 57,21 & 0,0167 \\
\hline 25 & & & & & & & & 11 & 87 & & \\
\hline 25 & & & & & & & & 9 & 70,3 & 50,5 & \\
\hline 30 & 198 & 2215 & 2 & 5235 & 1570 & 232 & 3650 & 14 & 81,9 & 56,06 & \\
\hline 32 & & & & & & & & 16 & 87,8 & 40,1 & 0,0085 \\
\hline
\end{tabular}

Cuadro 3. Puntuación para TF sobre la base del Factor de AGD.

\begin{tabular}{|c|c|c|}
\hline Código & Condición & Descripción \\
\hline A & Buena & DAF $<1.2$ \\
\hline B & Aceptable & $1.2 \leq \mathrm{DGAF}<1.5$ \\
\hline C & Necesaria precaución & $1.5 \leq \mathrm{DGAF}<2$ \\
\hline D & Pobre & $2 \leq \mathrm{DGAF}<3$ \\
\hline E & Muy pobre & $\mathrm{DGAF} \geq 3$ \\
\hline
\end{tabular}

Fuente: $[3,14]$

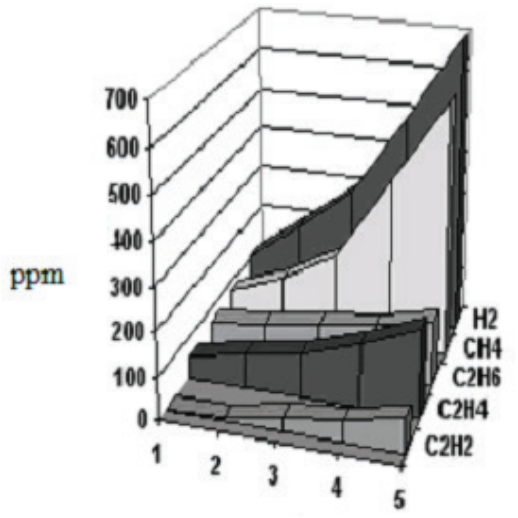

Puntuación

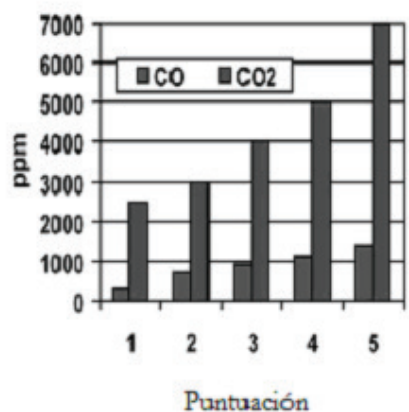

Puntuación

Figura 1. Puntuación según gases disueltos en el aceite. Fuente: [3] 
Del cálculo se obtuvieron los resultados que se muestran en el cuadro 4:

Cuadro 4. Factores utilizados para el cálculo del FAGD.

\begin{tabular}{|c|c|c|c|c|c|c|c|}
\hline & $\mathrm{H}_{2}$ & $\mathrm{CH}_{4}$ & $\mathrm{C}_{2} \mathrm{H}_{2}$ & $\mathrm{C}_{2} \mathrm{H}_{4}$ & $\mathrm{C}_{2} \mathrm{H}_{6}$ & $\mathrm{CO}$ & $\mathrm{CO} 2$ \\
\hline $\mathrm{S}$ & 5 & 6 & 1 & 6 & 3 & 1 & 5 \\
\hline $\mathrm{W}$ & 2 & 3 & 5 & 3 & 3 & 1 & 1 \\
\hline
\end{tabular}

De lo que se obtiene FAGD=3,66667 $\geq 3$, que, según el cuadro 3, es un resultado muy pobre, correspondiendo con la letra $\mathrm{E}$.

\section{Calidad del aceite}

Para la calidad del aceite se utiliza un método de puntuación, similar al que se aplica al AGD, que se muestra en el cuadro 5.

Del cálculo, para el TF que se toma de ejemplo en el cuadro 3, se obtienen los resultados siguientes: Fortaleza dieléctrica, kV/mm (2mm), $\mathrm{Si}=1$, Wi=3; Tensión interfacial (TIF), dinas/cm, $\mathrm{Si}=1, \mathrm{Wi}=2$; Acidez, mg KOH/g, $\mathrm{Si}=1, \mathrm{Wi}=1$; Agua, ppm, $\mathrm{Si}=1, \mathrm{Wi}=4$.

El factor de calidad del aceite FCA para este TF es FCA=1; es decir, el resultado clasifica como bueno (Ver cuadro 6).

Cuadro 5. Coeficientes según datos de ensayos del aceite de transformadores, sobre la base de la norma IEEE C57.637 ${ }^{\mathrm{TM}}-2015$.

\begin{tabular}{|c|c|c|c|c|}
\hline Un & $69 \mathrm{kV}<\mathrm{Un}<230 \mathrm{kV}$ & $230 \mathrm{kV} \leq \mathrm{Un}$ & $\mathrm{S}_{\mathrm{i}}$ & $W_{i}$ \\
\hline \multirow{4}{*}{ Fortaleza dieléctrica, $\mathrm{kV} / \mathrm{mm}$ (2mm) } & $\geq 52$ & $\geq 60$ & 1 & \multirow{4}{*}{3} \\
\hline & $47-52$ & $50-60$ & 2 & \\
\hline & $35-47$ & $40-50$ & 3 & \\
\hline & $\leq 35$ & $\leq 40$ & 4 & \\
\hline \multirow{4}{*}{ Tensión interfacial (TIF), dinas/cm } & $\geq 30$ & $\geq 32$ & 1 & \multirow{4}{*}{2} \\
\hline & $23-30$ & $25-32$ & 2 & \\
\hline & $18-23$ & $20-25$ & 3 & \\
\hline & $\leq 18$ & $\leq 20$ & 4 & \\
\hline \multirow[t]{4}{*}{ Acidez, mg KOH/g } & $\leq 0,04$ & $\leq 0,03$ & 1 & \multirow{4}{*}{1} \\
\hline & $0,04-0,1$ & $0,03-0,07$ & 2 & \\
\hline & $0,1-0,15$ & $0,07-0,1$ & 3 & \\
\hline & $\geq 0,15$ & $\geq 0,1$ & 4 & \\
\hline \multirow[t]{4}{*}{ Agua, ppm } & $\leq 20$ & $\leq 15$ & 1 & \multirow{4}{*}{4} \\
\hline & $20-25$ & $15-20$ & 2 & \\
\hline & $25-30$ & $20-25$ & 3 & \\
\hline & $\geq 30$ & $\geq 25$ & 4 & \\
\hline
\end{tabular}

Fuente: $[3,14,16,21]$.

Cuadro 6. Puntuación para TF sobre la base del FCA.

\begin{tabular}{|c|c|c|}
\hline A & Bueno & 1 \\
\hline B & Aceptable & 2 \\
\hline C & Necesaria precaución & 3 \\
\hline D & Pobre & 4 \\
\hline
\end{tabular}




\section{Derivados furánicos}

Los furanos se generan por la degradación del papel impregnado en aceite y se disuelven fácilmente en este; sus altas concentraciones o el incremento en estas indican la degradación del papel por el envejecimiento o fallas. Los furanos pueden causar el deterioro del papel si $\mathrm{T}<100^{\circ} \mathrm{C}$ y también cuando $\mathrm{T}=100 \ldots 200^{\circ} \mathrm{C}$ [2]. Se recomienda realizar el ensayo de derivados furánicos cuando el TF se sobrecalienta, cuando el contenido de $\mathrm{CO}$ o $\mathrm{CO} 2$ disueltos en el aceite es alto o si el plazo de servicio del equipo es mayor de 25 años [14]. El AGD del aceite de los TF se realiza mediante la cromatografía de gases, mientras que el análisis de furanos disueltos en el aceite requiere de la cromatografía de fase líquida. Estas técnicas difieren, por lo que se hacen necesarias, para la realización de las mismas, la utilización de equipamientos específicos en cada caso. En Cuba sólo se ha introducido la cromatografía de gases, pero la de fase líquida es aún inexistente. En los casos en que se ha requerido, ha sido necesario contratar este análisis para su realización en el extranjero [22]. Por esta razón, no se dispone de información sobre los derivados furánicos disueltos en el aceite del TF que se analiza, para la realización del cálculo de su IS.

\section{Cambiador de derivaciones}

En este tipo de TF no es posible tomar muestras de aceite para la realización de AGD en el aceite de este accesorio, por lo que esta variable tampoco puede incluirse en el cálculo.

\section{Información sobre mantenimiento}

Para calcular el IS se desarrolló un sistema de clasificación basado en las órdenes de trabajo (OT) emitidas en los últimos cinco años para el TF y sus accesorios. En esta evaluación son importantes la termografía infrarroja, la condición de los bushings, los salideros de aceite y su nivel, el estado del sistema de enfriamiento, las juntas, la condición del tanque principal (cuba), el aterramiento y otros factores [14]. Para realizar la selección de las calificaciones de los componentes individuales, de no existir ninguna OT sobre un componente dado en los últimos 5 años, se le otorga una calificación de "A".

El cuadro 7 muestra los factores de condición general que se introducen para incluir la tasa de OT emitidas en los últimos cinco años.

Cuadro 7. Condición general basada en la tendencia de OT totales emitidas en los últimos cinco años.

\begin{tabular}{|c|c|}
\hline $\begin{array}{l}\text { Puntuación según } \\
\text { condición }\end{array}$ & Descripción del criterio de condición \\
\hline A & [Máx. (últimos 2 años) < 3] O [incremento < 10\% sobre 5 años] \\
\hline B & $\begin{array}{l}\text { [Máx. (últimos } 2 \text { años) }>3 \text { E incremento }>10 \% \text { sobre } 5 \text { años] O [Máx. (últimos } 2 \\
\text { años) }>5 \text { ] }\end{array}$ \\
\hline C & $\begin{array}{l}\text { [Máx. (últimos } 2 \text { años) }>5 E \text { incremento }>30 \% \text { sobre } 5 \text { años] O [Máx. (últimos } 2 \\
\qquad \begin{array}{r}\text { años) }>10]\end{array}\end{array}$ \\
\hline $\mathrm{D}$ & $\begin{array}{l}\text { [Máx. (últimos } 2 \text { años) > } 10 \mathrm{E} \text { incremento }>50 \% \text { sobre } 5 \text { años] O [Máx. (últimos } 2 \\
\qquad \text { años) > 15] }\end{array}$ \\
\hline $\mathrm{E}$ & $\begin{array}{l}\text { [Máx. (últimos } 2 \text { años) > } 15 \mathrm{E} \text { incremento }>80 \% \text { sobre } 5 \text { años] O [Máx. (últimos } 2 \\
\text { años) > 20] }\end{array}$ \\
\hline
\end{tabular}

Fuente: [14]

De lo que se obtiene:

1. Condición general del TF-O OT-A;

2. Condición de bushings-4 OT-B; 
3. Condición del tanque principal-0 OT-A;

4. Sistema de enfriamiento-O OT-A;

5. Corrosión del tanque principal-0 OT-A;

6. Cimientos-O OT-A;

7. Aterramiento-0 OT-A;

8. Juntas-4 OT-B;

9. Conectores-O OT-A;

10. Salideros de aceite-4OT-B;

11. Nivel de aceite-2 OT-A;

12. Condición general del cambiador de derivaciones-4 OT-B.

\section{Cálculo final del Índice de Salud}

El cuadro 8 muestra la puntuación que se otorga en otros ensayos que se toman en cuenta para el cálculo.

Cuadro 8. Puntuación para los ensayos de relación de transformación y resistencia de enrollados a la corriente directa (CD).

\begin{tabular}{|c|c|c|}
\hline $\begin{array}{c}\text { Código de } \\
\text { puntuación }\end{array}$ & $\begin{array}{c}\text { Desviación de la relación de transformación } \\
\text { con respecto a la de fábrica, } \%\end{array}$ & $\begin{array}{c}\text { Desviación de la resistencia de enrollados } \\
\text { a la CD con respecto a la de fábrica, } \%\end{array}$ \\
\hline A & $\Delta T R \leq 0.1 \%$ & $\Delta R<1 \%$ \\
\hline$B$ & $0.1 \%<\Delta T R \leq 0.5 \%$ & $1 \% \leq \Delta R<2 \%$ \\
\hline C & $0.5 \%<\Delta T R \leq 1 \%$ & $2 \% \leq \Delta R<3 \%$ \\
\hline$D$ & $1 \%<\Delta T R<2 \%$ & $3 \% \leq \Delta R<5 \%$ \\
\hline$E$ & $\Delta T R \geq 2 \%$ & $\Delta R \geq 5 \%$ \\
\hline
\end{tabular}

Fuente: [14]

Los resultados del cálculo de $\Delta T R, \%$ se muestran en el cuadro 9, mientras en el cuadro 10 aparecen los resultados del cálculo de $\Delta \mathrm{R}, \%$.

Cuadro 9. Resultados del cálculo de $\Delta T R, \%$.

\begin{tabular}{|c|c|c|c|c|}
\hline Ramificación & Lugar de ensayo & AN/ab & BN/bc & CN/ca \\
\hline \multirow{2}{*}{9} & Fábrica & 10,688 & 10,703 & 10,698 \\
\cline { 2 - 5 } & Campo (último ensayo) & 10,779 & 10,749 & 10,754 \\
\cline { 2 - 5 } & $\Delta \mathrm{TR}, \%$ & 0,844234159 & 0,427946786 & 0,52073647 \\
\hline \multirow{2}{*}{10} & Fábrica & 10,519 & 10,524 & 10,518 \\
\cline { 2 - 5 } & Campo (último ensayo) & 10,596 & 10,572 & 10,575 \\
\cline { 2 - 5 } & $\Delta \mathrm{TR}, \%$ & 0,726689317 & 0,454029512 & 0,539007092 \\
\hline
\end{tabular}


Cuadro 10. Resultados del cálculo de $\Delta R, \%$.

\begin{tabular}{|c|c|c|c|c|}
\hline Ramificación & Lugar de ensayo & AN & BN & CN \\
\hline \multirow{4}{*}{9} & Fábrica & 0,54410 & 0,56600 & 0,54980 \\
\cline { 2 - 5 } & Campo (último ensayo) & 0,558 & 0,560 & 0,567 \\
\cline { 2 - 5 } & $\Delta R, \%$ & 2,491039427 & 1,060070671 & 3,0335097 \\
\hline \multirow{3}{*}{10} & Fábrica & 0,52440 & 0,53660 & 0,52999 \\
\cline { 2 - 5 } & Campo (último ensayo) & 0,539 & 0,540 & 0,546 \\
\cline { 2 - 5 } & $\Delta R, \%$ & 2,708719852 & 0,62962963 & 2,932234432 \\
\hline
\end{tabular}

De cada tipo de desviación se escogen los mayores valores. Se toman $\Delta T R=0,844234159 \%$, con código de puntuación $\mathrm{C} ; \Delta R=3,0335097$, con código de puntuación $\mathrm{D}$.

El total de ensayos para el cálculo del IS se generaliza en el cuadro 11. Las dos columnas de la derecha indican las puntuaciones en concreto para cada componente del TF.

Tomando en consideración los componentes mostrados y mediante la realización de un cálculo por la ecuación (1) se obtuvo: IS= 0,687755102=68,77\%,

Esto, según el cuadro 1, indica que el TF en cuestión puede estar fallado, lo que corresponde con su comportamiento durante su explotación, por lo que se recomienda Incrementar los ensayos de diagnóstico que se realizan al equipo.

En el cuadro 12 se introducen los datos del TF de menor edad en la flota, a fin de calcular también su IS y poder comprobar si el método empleado permite realizar un cálculo correcto.

Cuadro 11. Puntuación para el IS.

\begin{tabular}{|c|c|c|c|c|c|c|}
\hline$\#$ & Factor de diagnóstico & K & $\begin{array}{l}\text { Posibles puntuaciones de } \\
\text { condición }\end{array}$ & $\begin{array}{l}\text { Posibles factores } \\
\text { de IS }\end{array}$ & $\begin{array}{l}\text { Condición } \\
\text { real }\end{array}$ & $\begin{array}{l}\text { Factor real } \\
\text { de IS }\end{array}$ \\
\hline 1 & AGD & 10 & $A, B, C, D, E$ & $4,3,2,1,0$ & $E$ & 0 \\
\hline 2 & Calidad del aceite & 6 & $A, B, C, D, E$ & $4,3,2,1,0$ & A & 4 \\
\hline 3 & Condición general del TF & 8 & $A, B, C, D, E$ & $4,3,2,1,0$ & A & 4 \\
\hline 4 & Relación de transformación & 5 & $A, B, C, D, E$ & $4,3,2,1,0$ & C & 2 \\
\hline 5 & $\begin{array}{l}\text { Resistencia de los enrollados a } \\
\qquad \text { la CD }\end{array}$ & 6 & $A, B, C, D, E$ & $4,3,2,1,0$ & $\mathrm{D}$ & 1 \\
\hline 6 & Condición de bushings & 5 & $A, B, C, D, E$ & $4,3,2,1,0$ & B & 3 \\
\hline 7 & Corrosión del tanque principal & 2 & $A, B, C, D, E$ & $4,3,2,1,0$ & A & 4 \\
\hline 8 & Sistema de enfriamiento & 2 & $A, B, C, D, E$ & $4,3,2,1,0$ & A & 4 \\
\hline 9 & $\begin{array}{l}\text { Corrosión del tanque } \\
\text { expansionador }\end{array}$ & 1 & $A, B, C, D, E$ & $4,3,2,1,0$ & A & 4 \\
\hline 10 & Cimientos & 1 & $A, B, C, D, E$ & $4,3,2,1,0$ & A & 4 \\
\hline 11 & Aterramiento & 1 & $A, B, C, D, E$ & $4,3,2,1,0$ & A & 4 \\
\hline 12 & Juntas & 1 & $A, B, C, D, E$ & $4,3,2,1,0$ & $B$ & 3 \\
\hline 13 & Conectores & 1 & $A, B, C, D, E$ & $4,3,2,1,0$ & A & 4 \\
\hline 14 & Salideros de aceite & 1 & $A, B, C, D, E$ & $4,3,2,1,0$ & B & 3 \\
\hline 15 & Nivel de aceite & 1 & $A, B, C, D, E$ & $4,3,2,1,0$ & A & 4 \\
\hline 16 & $\begin{array}{l}\text { Condición general del } \\
\text { cambiador de derivaciones }\end{array}$ & 5 & $A, B, C, D, E$ & $4,3,2,1,0$ & B & 3 \\
\hline
\end{tabular}

Fuente: [14] 
Cuadro 12. Datos de segundo TF para cálculo de su IS.

\begin{tabular}{|c|c|c|c|c|c|c|c|c|c|c|c|}
\hline & \multicolumn{9}{|c|}{ Gases disueltos, ppm } & \multicolumn{3}{c|}{ Características dieléctricas del aceite aislante } \\
\hline $\begin{array}{c}\text { Años de } \\
\text { servicio }\end{array}$ & $\mathrm{H}_{2}$ & $\mathrm{CH}_{4}$ & $\mathrm{C}_{2} \mathrm{H}_{2}$ & $\mathrm{C}_{2} \mathrm{H}_{4}$ & $\mathrm{C}_{2} \mathrm{H}_{6}$ & $\mathrm{CO}$ & $\mathrm{CO}_{2}$ & $\begin{array}{c}\text { Agua, } \\
\mathrm{ppm}\end{array}$ & $\begin{array}{c}\text { Tensión } \\
\text { de } \\
\text { ruptura, } \\
\mathrm{kV}\end{array}$ & $\begin{array}{c}\text { Tensión } \\
\text { interfacial } \\
\text { (TIF), dinas/ } \\
\mathrm{cm}\end{array}$ & $\begin{array}{c}\text { Acidez, mg } \\
\mathrm{KOH} / \mathrm{g}\end{array}$ \\
\hline 3 & 37 & 2 & 2 & 2 & 2 & 1403 & 6033 & 11 & 91,2 & 48,73 & 0,0057 \\
\hline 4 & 12 & 14 & 2 & 2 & 2 & 1379 & 8266 & & 94,1 & 44,64 & 0,0154 \\
\hline 5 & 10 & 16 & 2 & 2 & 2 & 1415 & 8657 & & 55,8 & 47,78 & \\
\hline 6 & 5 & 18 & 2 & 2 & 2 & 1511 & 9404 & 12 & 94,2 & 43,27 & 0,0115 \\
\hline 7 & 10 & 19 & 2 & 2 & 2 & 1604 & 10015 & & & & \\
\hline 8 & & & & & & & & 7 & 84,7 & 33,05 & 0,0129 \\
\hline
\end{tabular}

Calculando se obtienen:

FAGD $=1,333333333$, lo que, según el cuadro 3 , es un valor aceptable $(1.2 \leq \mathrm{DGAF}<1.5)$, con un coeficiente de $B$.

Para el cálculo del FCA se obtienen: Fortaleza dieléctrica, kV/mm (2mm), Si=1, Wi=3; Tensión interfacial (TIF), dinas/cm, $\mathrm{Si}=1, \mathrm{Wi}=2$; Acidez, $\mathrm{mg} \mathrm{KOH/g}, \mathrm{Si}=1$, Wi=1; Agua, ppm, $\mathrm{Si}=1$, Wi=4.

El factor de calidad del aceite FCA para este TF es $F C A=1$; es decir, el resultado clasifica como bueno, con coeficiente A.

Total de OT por componentes en los últimos cinco años:

1. Condición general del TF-O OT-A;

2. Condición de bushings-0 OT-A;

3. Condición del tanque principal-0 OT-A;

4. Sistema de enfriamiento-3 OT-B;

5. Corrosión del tanque principal-0 OT-A;

6. Cimientos-0 OT-A;

7. Aterramiento-0 OT-A;

8. Juntas-0 OT-A;

9. Conectores-O OT-A;

10. Salideros de aceite-O OT-A;

11. Nivel de aceite-2 OT-A;

12. Condición general del cambiador de derivaciones-0-no cuenta con cambiador de derivaciones bajo carga.

Para el cálculo de $\Delta T R, \%$ y $\Delta R$, \% se realiza el mismo procedimiento que para el TF que se mostró en el cuadro 2, escogiendo los mayores valores entre los tres posibles para cada uno; por tanto, se tomaron $\Delta T R=0,522778193 \%$, con código de puntuación $C$ y $\Delta R=5,69105691$, con código de puntuación $\mathrm{E}$. 
Para el cálculo total:

\begin{tabular}{|c|c|c|c|c|}
\hline$\#$ & Factor de diagnóstico & K & Condición & Factor de IS \\
\hline 1 & AGD & 10 & B & 3 \\
\hline 2 & FCA & 6 & A & 4 \\
\hline 3 & Condición general del TF & 8 & A & 4 \\
\hline 4 & Relación de transformación & 5 & C & 2 \\
\hline 5 & Resistencia de los enrollados a la CD & 6 & E & 0 \\
\hline 6 & Condición de bushings & 5 & A & 4 \\
\hline 7 & Corrosión del tanque principal & 2 & A & 4 \\
\hline 8 & Sistema de enfriamiento & 2 & B & 3 \\
\hline 9 & Corrosión del tanque expansionador & 1 & A & 4 \\
\hline 10 & Cimientos & 1 & A & 4 \\
\hline 11 & Aterramiento & 1 & A & 4 \\
\hline 12 & Juntas & 1 & A & 4 \\
\hline 13 & Conectores & 1 & A & 4 \\
\hline 14 & Salideros de aceite & 1 & A & 4 \\
\hline 15 & Nivel de aceite & A & 4 \\
\hline
\end{tabular}

IS $=0,774509804=77,45098039 \%$.

Esto, según el cuadro 1, indica que el TF en cuestión está en buena condición, recomendándose para el mismo la realización de Mantenimiento normal.

\section{Resultados y futuros trabajos}

Los resultados obtenidos del cálculo del IS para cada TF muestran correspondencia con el estado general de cada uno y con la opinión de expertos consultados. Para realizar el cálculo del IS de un parque de TF se utilizan poderosas herramientas matemáticas, que no serán tratadas en el presente trabajo, constituyendo objeto de investigaciones futuras.

\section{Conclusiones}

En el artículo se comprobó que el método propuesto para el cálculo del IS en $[3,14,16]$ puede utilizarse en las condiciones de Cuba, donde no se han implementado numerosas técnicas de ensayo, debido a la imposibilidad de adquisición de equipos para su realización. En futuros trabajos se definirán herramientas matemáticas más amplias para la realización del cálculo del IS de flotas de TF en las condiciones de nuestro país.

\section{Referencias}

[1] E. Calo, R. E. Álvarez, P. Morcelle del Valle, and L. Catalano, "Análisis de IEEE C57.152 y TB 445 de CIGRE para el diagnóstico de la condición de transformadores de potencia," in XVII Encuentro Regional Iberoamericano (ERIAC) de CIGRÉ Ciudad del Este, Paraguay 2017, vol. Comité de Estudio CE A2-Transformadores p. 8: CIGRÉ, 2017.

[2] A. Teymouri and B. Vahidi, "CO2/CO concentration ratio: A complementary method for determining the degree of polymerization of power transformer paper insulation," (in English), IEEE Electrical Insulation Magazine, vol. 33, no. 1, pp. 24-30, January-February 20172017.

[3] B. Gorgan, P. V. Notingher, L. V. Badicu, and G. Tanasescu, "Calculation of power transformers health indexes," (in English), Annals of the University of Craiova, vol. Electrical Engineering series, no. 34, pp. 13-18, 2010. 
[4] J. J. Montané García, D. D. S. A., and A. M. del Castillo Serpa, "Avances en evaluación del estado y la vida remanente de transformadores de fuerza," (in Español), Ingeniería energética, vol. 40, no. 3, pp. 245-256, 2019.

[5] S. M. Akolkar and B. E. Kushare, "Remaining Life Assessment of Power Transformer," (in English), Journal of Automation and Control, vol. 2, no. 2, p. 4, 2014.

[6] E. Abbasi and O. P. Malik, "Failure rate estimation of power transformers using inspection data," presented at the 2016 International Conference on Probabilistic Methods Applied to Power Systems (PMAPS), Beijing, China 16-20 Oct. 2016, 2016. Available: https://ieeexplore.ieee.org/document/7764150/

[7] B. P. Das and L. Cheim, "Transformer assessment using health index-Part 1-Sensitivity analys and critical discussion " (in English), Transformer Magazine, vol. 7, no. 4, pp. 42-49, October 20202020.

[8] E. Abbasi, "Developing Practical Methods for Ageing and Failure Probability Modeling of Mineral Oil Immersed Power Transformers Using Smart Utility Data," Thesis in order to obtain the degree of Doctor of Philosophy, Department of Electrical and Computer Engineering, University of Calgary Calgary, Alberta, Canada, 2017.

[9] J. A. Tariacuri Q., G. Aponte M., and H. Cadavid R., "Indice de salud para transformadores de potencia usando el analisis de gases disueltos y 2-Fal," (in Espanol), e-Gnosis vol. 18, no. 1, pp. 1-15, January 20202020.

[10] G. Aponte M. et al., "Herramienta de software para estimar el índice de salud en transformadores de potencia," e-Gnosis vol. 18, no. 10, pp. 1-21, Septiembre 18, 2020 Accessed on: 29 de julio del 2020Available: http:/l www.e-gnosis.udg.mx/index.php/e-gnosis/article/view/793/414

[11] J. C. Parraga Acosta, "El índice de salud y su influencia en la gestión del ciclo de vida de los transformadores del sistema eléctrico de la Mina Cobriza " Tesis para optar por el grado académico de Maestro en Ciencias, Mención Sistemas Eléctricos de Distribución, Facultad de Ingeniería Eléctrica y Electrónica Universidad de Nacional del Centro del Perú Huancayo, Perú 2019

[12] S. Li, G. Wu, H. Dong, L. Yang, and X. Zhen, "Probabilistic Health Index-Based Apparent Age Estimation for Power Transformers," (in English), IEEE Access, vol. 8, pp. 9692-9701, January 20202020.

[13] S. Milosavljević and A. Janjić, "Power transformer health index estimation using evidential reasoning," (in English), FACTA UNIVERSITATIS vol. 33, no. 4, pp. 571-581, December 20202020.

[14] A. N. Jahromi, R. Piercy, S. Cress, J. R. R. Service, and W. Fan, "An Approach to Power Transformer Asset Management Using Health Index " (in English), IEEE Electrical Insulation Magazine vol. 25, no. 2, March-April 2009, pp. 20-34, 21 March 20092009.

[15] I. G. N. Satriyadi Hernanda, A. C. Mulyana, D. A. Asfani, I. M. Y. Negara, and D. Fahmi, "Application of Health Index Method for Transformer Condition Assessment " presented at the IEEE TENCON2014, Bangkok, Thailand, October 2014, 2014. Available: https://www.researchgate.net/publication/279913935 Application of Health Index Method for Transformer Condition Assessment

[16] G. Tanasescu et al., "Assessment of Power Transformers Conditions Based on Health Index," presented at the CIGRÉ Regional South-East European Conference (RSEEC 2012) Hotel Hilton, Sibiu, Romania, October 2012, 2012. Available: https://www.researchgate.net/publication/266732201_Assessment_of_Power_Transformers Conditions Based on Health Index

[17] Z. D. Wang et al., "Ageing Assessment of Transformers through Oil Test Database Analyses and Alternative Diagnostic Techniques " presented at the 2015 CIGRE SC A2 Colloquium, Shanghai, China, September 2015, 2015. Available: https://www.researchgate.net/publication/282245702 Ageing Assessment of Transformers through_Oil_Test_Database_Analyses_and_Alternative_Diagnostic_Techniques

[18] Mineral insulating oils in electrical equipment-Supervision and maintenance guidance, 2013.

[19] Mineral oil-impregnated electrical equipment in service - Guide to the interpretation of dissolved and free gases analysis, ICS 17.220.99; 29.040.10; 29.180, 2015.

[20] IEEE Guide for the Interpretation of Gases Generated in Mineral Oil-Immersed Transformers 2019

[21] IEEE Guide for the Reclamation of Mineral Insulating Oil and Criteria for Its Use, 2015.

[22] J. J. Montané García, S. A. Dorrbercker Drake, and A. M. del Castillo Serpa, "Uso de relaciones químicas en cálculo de tiempo de vida útil y remanente de transformadores de fuerza," presented at the XIV Congreso Internacional de Alta Tensión y Aislamiento Eléctrico ALTAE 2019, Guadalajara, Jalisco, México, 30 de octubre del 2019, 2019. Available: https://www. researchgate.net/publication/338233360 USO DE RELACIONES QUIMICAS EN_CALCULO_DE_TIEMPO DE_VIDA_UTIL_Y REMANENTE_DE_TRANSFORMADORES_DE_ FUERZA 